\title{
Autologous Co-culture of Primary Human Alveolar Macrophages and Epithelial Cells for Investigating Aerosol Medicines. Part I: Model Characterisation
}

\author{
Marius Hittinger, ${ }^{1,2}$ Julia Janke, ${ }^{3}$ Hanno Huwer, ${ }^{4}$ Regina Scherließ, ${ }^{3}$ Nicole Schneider-Daum² \\ and Claus-Michael Lehr1,2,5
}

${ }^{1}$ PharmBioTec GmbH, Saarbrücken, Germany; ${ }^{2}$ Department of Drug Delivery, Helmholtz Institute for Pharmaceutical Research Saarland, Saarbrücken, Germany; ${ }^{3}$ Department of Pharmaceutics and Biopharmaceutics, Christian Albrecht University Kiel, Kiel, Germany; ${ }^{4}$ Heart \& Thoracic Surgery, SHG Kliniken Völklingen, Saarbrücken, Germany; ${ }^{5}$ Biopharmaceutics and Pharmaceutical Technology, Department of Pharmacy, Saarland University, Saarbrücken, Germany

\begin{abstract}
Summary - The development of new formulations for pulmonary drug delivery is a challenge on its own. New in vitro models which address the lung are aimed at predicting and optimising the quality, efficacy and safety of inhaled drugs, to facilitate the more rapid translation of such products into the clinic. Reducing the complexity of the in vivo situation requires that such models reproducibly reflect essential physiological factors in vitro. The choice of cell types, culture conditions and the experimental set-up, can affect the outcome and the relevance of a study. In the alveolar space of the lung, epithelial cells and alveolar macrophages are the most important cell types, forming an efficient cellular barrier to aerosols. Our aim was to mimic this barrier with primary human alveolar cells. Cell densities of alveolar macrophages and epithelial cells, isolated from the same human donor, were optimised, with a focus on barrier properties. The combination of 300,000 epithelial cells $/ \mathrm{cm}^{2}$ together with 100,000 macrophages $/ \mathrm{cm}^{2}$ showed a functional barrier (transepithelial electrical resistance $>500 \Omega . \mathrm{cm}^{2}$ ). This cell model was combined with the Pharmaceutical Aerosol Deposition Device on Cell Cultures. The functionality of the in vitro system was investigated with spray-dried fluorescently labelled poly(lactic-co-glycolic) acid particles loaded with ovalbumin as a model drug.
\end{abstract}

Key words: advanced in vitro model, lung model, PADDOCC, PLGA, pulmonary drug delivery, Shuttle \& Find.

Address for correspondence: Nicole Schneider-Daum, Department of Drug Delivery, Helmholtz Institute for Pharmaceutical Research Saarland, Saarbrücken, Germany.

E-mail: nicole.daum@helmholtz-hzi.de

\section{Introduction}

The alveolar space is characterised by a high surface area $\left(\sim 100 \mathrm{~m}^{2}\right)$ and a thin cellular barrier. Inhaled airborne particles with a small aerodynamic diameter are preferentially deposited in the alveolar space (1). In order to predict interactions of inhaled drug products with the respiratory tract, in vitro models have to be able to mimic certain aspects of the healthy and the diseased human lung (2). Depending on their effective development, characterisation and validation, they could serve as powerful tools to estimate possible dosage ranges, uptake behaviour or inflammatory responses, while also minimising or completely avoiding animal use, in line with the Three Rs principles of Russell and Burch. Apart from innovative lung-on-a-chip approaches, such as those developed by Huh et al. (3), the central component of most respiratory tract models is a static Transwell ${ }^{\mathrm{TM}}$ membrane or a similar system. The models can comprise several different cell types, either maintained under submerged conditions (when the cells are covered with medium) or at the air-liquid interface.

Physiologically relevant air-liquid interface conditions permit the combination of the cell cultures with aerosol deposition devices, such as, for example, the VITROCELL Cloud System ${ }^{\text {TM }}$ (4) or the Pharmaceutical Aerosol Deposition Device on Cell Cultures (PADDOCC; 5). Furthermore, these conditions permit the study of the effects related to the deposition of aerosol particles in specific lung regions. The PADDOCC was developed to mimic the in vivo deposition of dry powder formulations, and it can be combined with commercially-available dry powder inhaler-devices (e.g. the Handihaler ${ }^{\mathrm{TM}}$ ). With this system, only the respirable particle fraction is deposited on the cells by sedimentation, thus reflecting key aspects of pulmonary drug delivery.

Isolated human primary alveolar type (AT) II cells can be differentiated to AT I-like cells, which display important in vivo characteristics, such as 
barrier properties and a large cellular surface (6, 7). The differentiation from type II to type I includes the formation of tight junctions associated with changes in morphology (from cuboidal to flat), and an increased bioelectric resistance (7). In addition, the secretion of surfactant proteins (such as SP-C) decreases, which was shown at the mRNA and protein levels, and the type I-associated expression of aquaporin- 5 and caveolin-1 increases (6).

During the isolation of cells from lung biopsies, human alveolar macrophages are usually removed, and are eventually used for other purposes (8), while the purified epithelial cells are grown to confluent tight monolayers (9). Rather than discarding the isolated macrophages, the aim of this study was to recombine them with the epithelial cell monolayers obtained from the same biopsy, and thus establish a co-culture based on human primary alveolar epithelial cells and macrophages from the same donor (i.e. autologous co-culture). In the first step, both cell types were combined and maintained as a submerged culture. Subsequently, the co-culture was adjusted to air-liquid interface conditions, in order to mimic the in vivo situation in the deep lung, thereby allowing aerosol deposition in combination with the PADDOCC. A dry powder formulation based on poly(lactide-co-glycolide) acid (PLGA) nanoparticles in a microparticulate matrix was then applied as part of the preliminary pharmaceutical evaluation of such a human autologous co-culture.

\section{Materials and Methods}

\section{Isolation of primary alveolar macrophages and epithelial cells}

Lung tissue, from patients undergoing lung resection, was received from the SHG Kliniken Völklingen, with the formal consent of the local ethics committee (State Medical Board of Registration, Saarland, Germany). The epithelial cells were isolated according to an established protocol (9), on tissues from different donors. In brief, lung tissue was chopped into small pieces of $5 \mu \mathrm{m}$ edge-length and washed three times in balanced salt solution (137mM NaCl, 5mM KCl, 0.7mM Na${ }_{2} \mathrm{HPO}_{4}, 10 \mathrm{mM}$ HEPES, 5.5mM glucose, $\mathrm{pH} 7.4$ ) with a cell strainer $(100 \mu \mathrm{m}$ pore size; Becton Dickinson, Heidelberg, Germany). The first filtrate contained a high concentration of alveolar macrophages and was kept at room temperature for the subsequent macrophage isolation. The lung suspension was digested by incubating with a solution of $1.5 \mathrm{ml}$ trypsin $+300 \mu \mathrm{l}$ elastase $(10 \mathrm{mg} / \mathrm{ml}$ stock) (Sigma-Aldrich, Deisenhofen, Germany; Worthington, Lakewood, USA, respec- tively) for 40 minutes at $37^{\circ} \mathrm{C}$. The cells were subsequently incubated in cell culture Petri dishes for 90 minutes at $37^{\circ} \mathrm{C}$, to remove tissue macrophages, and any remaining erythrocytes were removed with a Percoll gradient (Sigma-Aldrich). The alveolar type II cells were isolated from the cell suspension by positive selection with an EpCAM antibody (Miltenyi Biotec, Bergisch Gladbach, Germany). The cells were seeded on Transwell/Snapwell ${ }^{\mathrm{TM}}$ membranes with a pore size of $0.4 \mu \mathrm{m}$ (Corning Costar, Bodenheim, Germany), that were pre-coated with a mixture of fibronectin (Becton Dickinson) and collagen (Sigma-Aldrich) according to the following procedure: A $50 \mu \mathrm{l}$ aliquot of collagen $(3 \mathrm{mg} / \mathrm{ml}$ stock) and a $50 \mu \mathrm{l}$ aliquot of fibronectin $(1 \mathrm{mg} / \mathrm{ml}$ stock) were added to $5 \mathrm{ml}$ sterile filtered distilled water. A $200 \mu \mathrm{l}$ portion of the mixture was added to the apical compartment of the $0.33 \mathrm{~cm}^{2}$ Transwell inserts $(500 \mu \mathrm{l}$ in the case of the $1.12 \mathrm{~cm}^{2}$ inserts). The Transwells were incubated for 90 minutes at $37^{\circ} \mathrm{C}$ and the collagen-fibronectin mixture was removed. The filters were dried for 10 minutes under sterile conditions. The AT II cells were cultured in Small Airway Epithelial Cell Growth Medium (SAGM ${ }^{\mathrm{TM}}$; Lonza, Verviers, Belgium).

For the isolation of alveolar macrophages, the filtrate of the first washing step, comprising mainly erythrocytes and alveolar macrophages, was used. The cells were washed with RPMI medium (Gibco, Darmstadt, Germany) containing $5 \% \mathrm{v} / \mathrm{v}$ fetal calf serum (FCS) and $1 \% \mathrm{v} / \mathrm{v}$ penicillin/streptomycin (P/S). Subsequently, the cells were distributed onto three cell culture Petri dishes and incubated for one hour $\left(37^{\circ} \mathrm{C}, 5 \% \mathrm{CO}_{2}\right.$, $100 \%$ humidity). The (non-adherent) erythrocytes were removed from the (adherent) alveolar macrophages by washing three times with buffered salt solution. After the final wash, the buffer was replaced by RPMI. The medium was renewed after the first 24-hour period and the cells were cultured until day 4 (starting from day 0 as day of isolation).

\section{Optimisation of cell seeding densities}

Tight junctions were stained by incubating overnight at $4^{\circ} \mathrm{C}$ with an anti-occludin antibody (Invitrogen, Carlsbad, CA, USA) diluted 1:200 in PBS containing $1 \% \mathrm{v} / \mathrm{v}$ bovine serum albumin (BSA). Subsequently, a FITC-labelled secondary antibody (Santa Cruz Biotechnology, Dallas, TX, USA), diluted 1:250 in PBS containing 1\% v/v BSA, was added, and the samples were incubated for 1.5 hours at room temperature. The transepithelial electrical resistance (TEER) was measured with an EVOM $^{\mathrm{TM}}$ (World Precision Instruments, Sarasota, FL, USA).

Human alveolar epithelial cells were seeded at different cell densities $\left(50\right.$ cells $/ \mathrm{cm}^{2}, 100$ cells $/ \mathrm{cm}^{2}$, 
200 cells $/ \mathrm{cm}^{2}, 300$ cells $/ \mathrm{cm}^{2}$ and 600,000 cells $/ \mathrm{cm}^{2}$ ) on Transwell inserts (Cat. No. 3470, $0.33 \mathrm{~cm}^{2}$; Corning, New York, NY, USA), and cultured for up to eight days. The TEER was measured with an EVOM every day, and the maximum TEER was plotted as a function of cell density. Three cell isolations were used and for each measurement point the mean values for at least four Transwell inserts were determined.

On day four, different densities of macrophages were added on top of the epithelial cells $(30,000$ cells $/ \mathrm{cm}^{2} ; 60,000$ cells $/ \mathrm{cm}^{2} ; 100,000$ cell $\mathrm{s} / \mathrm{cm}^{2} ;$ and 300,000 cells $/ \mathrm{cm}^{2}$ ), with 0 cells $/ \mathrm{cm}^{2}$ representing the control sample. The macrophages were added in RPMI medium containing $5 \%$ v/v FCS and $1 \%$ $\mathrm{v} / \mathrm{v} \mathrm{P} / \mathrm{S}$. Therefore, the epithelial cells were in contact with SAGM in the basolateral compartment and with RPMI in the apical compartment. Figure 1 shows the scheme for the preparation of the human autologous co-culture. A TEER value > $300 \Omega . \mathrm{cm}^{2}$ was considered crucial before the addition of macrophages. The influence of macrophage density on barrier function was measured on day 5. Light microscopy was used to visualise the distribution of macrophages on the epithelial cell layer.

\section{Preparation and characterisation of the spray-dried PLGA nano-in-microparticles}

Fluoresceinamine isomer I (FA; Sigma-Aldrich, St Louis, MO, USA) was covalently bound to PLGA (Resomer $^{\circledR}$ RG 503H; Evonik, Darmstadt, Germany), according to the method described by Weiss et al. (10). After freeze-drying of the polymer, the FA-PLGA was used for the formation of nanoparticles. The nanoparticles were produced by a double emulsion technique, according to that of Semete et al. (11). For this, 2.5\% (w/v) FA-PLGA was dissolved in ethyl acetate (Merck, Darmstadt, Germany) as the organic phase. This organic phase was homogenised for two minutes at 20,500rpm with a Ultra-Turrax (IKA, Staufen, Germany), with a hydrophilic phase containing $4 \%(\mathrm{w} / \mathrm{v})$ ovalbumin (OVA; Sigma-Aldrich, USA) in phosphate buffered solution $\mathrm{pH}$ 7.4, resulting in a W/O emulsion. Afterwards, a second hydrophilic phase, containing 5\% (w/v) PVA (Mowiol 4-88; Hoechst, Frankfurt, Germany) dissolved in ultrapure water, was added and homogenised for two minutes. The resulting $\mathrm{W} / \mathrm{O} / \mathrm{W}$ emulsion was transferred into a stabilising solution with $1 \% \mathrm{w} / \mathrm{v}$ PVA in water, and stirred with a magnetic stirrer (IKA, Staufen, Germany) at 400rpm to evaporate the ethyl acetate. The dispersion was then washed to remove free ovalbumin. For this, the dispersion was centrifuged at 14,000rpm (23,000g) (Centrifuge 5430 R; Eppendorf, Hamburg, Germany), which resulted in a clear supernatant. The supernatant, containing free ovalbumin, was removed and replaced with a fresh $1 \%(w / v)$ PVA solution. The dispersion was spray-dried with the MiniBüchi B-290 (Büchi, Flawil, Switzerland), equipped with a two-fluid nozzle and a high-performance cyclone. No further matrix excipients were added prior to spray-drying. Drying was performed at an inlet temperature of $100^{\circ} \mathrm{C}$ and an outlet temperature of about $39^{\circ} \mathrm{C}$, to embed PLGA nanoparticles in a microparticulate matrix of PVA.

In order to determine the fine particle fraction (FPF), the dry powder was characterised with the Next Generation Impactor (NGI; Copley Scientific,

Figure 1: A scheme for the co-culture protocol

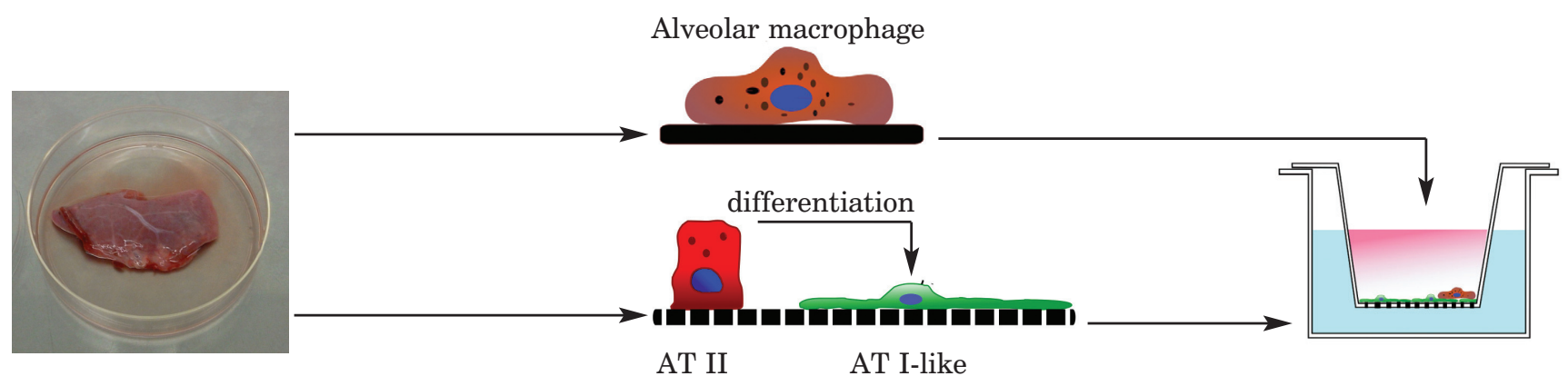

Day 0: isolation

Day 0-4: differentiation

Day 4: co-culture

After isolation, the cells were cultured as monolayers. Epithelial cells were differentiated on Transwell membranes from alveolar type II cells (AT II) to alveolar type I-like cells (AT I-like) until day four. Macrophages were then added and further cultured until day five. 
Nottingham, UK). The spray-dried powder was transferred into HPMC capsules of size 3 (Qualicaps Europe, Madrid, Spain), with 20mg/ capsule. The capsules were placed in the Cyclohaler (Pb Pharma GmbH, Meerbusch, Germany) and analysed with the NGI (coating = propylene glycol and isopropanol 50:50) at a flow rate of $100 \mathrm{~L} / \mathrm{min}$ under controlled environmental conditions $\left(21^{\circ} \mathrm{C}\right.$ and $45 \%$ relative humidity). The deposited powder was dissolved in $0.1 \mathrm{M} \mathrm{NaOH}$. After neutralisation with $0.1 \mathrm{M} \mathrm{HCl}$, the ovalbumin content of the samples $(n=4)$ was analysed with the Micro BCA Protein Assay Kit (Thermo Scientific, Rockford, IL, USA). The FPF and the mass median aerodynamic diameter (MMAD) of the delivered dose were calculated by using the Copley Inhaler Testing Data Analysis Software (CITDAS, Version 3.10; Copley Scientific Ltd, Nottingham, UK).

\section{Deposition of airborne PLGA particles on the co-culture with the PADDOCC}

For deposition of the spray-dried particles, the coculture was combined with the PADDOCC. This system had been previously optimised for Transwell membranes, with a surface area of $0.33 \mathrm{~cm}^{2}$. For this study, optimisation had to be extended to include Snapwell membranes, with a surface area of $1.12 \mathrm{~cm}^{2}$. Furthermore, the use of the PADDOCC had to be modified to accommodate the change from submerged to air-liquid interface culture conditions. This was because the poor adherence of macrophages to epithelial cells meant that air-liquid conditions could not be achieved by simply removing the medium from the apical compartment, as this would result in significant loss of macrophages. Therefore, the macrophages were concentrated by centrifugation, after counting with a Neubauer chamber, and were placed in an insert in $6 \mu \mathrm{l}$ culture medium volume on top of the epithelial cells under air-liquid conditions. The coculture was then incubated overnight $\left(37^{\circ} \mathrm{C}, 5 \%\right.$ $\mathrm{CO}_{2}, 100 \%$ humidity).

The PADDOCC was used according to an established protocol (11). In brief, spray-dried FA-PLGA particles were transferred into HPMC size 3 capsules (Qualicaps Europe, Madrid, Spain; 20mg/capsule) and placed in the HandiHaler. After activation, a dispersion impulse with a flow rate of $60 \mathrm{~L} / \mathrm{min}$ was induced for 0.2 seconds to aerosolise the powder. The ventilation flow transports the particles to the deposition unit within 2 seconds. The device was then switched off for 10 minutes, and the settings were changed to the deposition mode. The aerosol was deposited onto the Snapwells by sedimentation, then the revolver was turned to the ventilation mode. This process was repeated three times, in order to completely empty the capsule.
FA-labelled PLGA particles were aerosolised in the PADDOCC. The amount of particles deposited was determined in empty Snapwells. The particles deposited were dissolved in $1 \mathrm{ml}$ PBS, followed by quantification with a Tecan plate reader (Tecan Group Ltd, Maennedorf, Switzerland). In order to compare the particle size of the dry powder formulation before and after deposition in the PADDOCC, conductive SEM tabs were put into the Snapwells and the aforementioned deposition procedure was conducted. Correlative light and electron microscopy ensured that the labelling process of the particles was successful and that the deposition of the particles in the PADDOCC and on the cell culture could be visualised. The correlation was performed by using Shuttle \& Find ${ }^{\mathrm{TM}}$ (S\&F; Zeiss, Jena, Germany). S\&F includes a sample holder and a software package that permits the combination of different microscopes (12).

Co-cultures and monocultures were exposed to FA-PLGA particles on day five, as described above for cell-free wells in the PADDOCC. The TEER was measured after 24 hours of incubation. An MTT assay was performed on the cells in the inserts, according to the manufacturer's instructions (Sigma-Aldrich), with the exception that $300 \mu l$ of DMSO were added to the apical Transwell compartment, from which $200 \mu$ l were subsequently transferred to a 96 -well plate before the absorbance was measured. The deposition procedure was repeated on the same day and, instead of an MTT assay, the cells were fixed for correlative microscopy, 24 hours after particle deposition in the PADDOCC.

\section{Fixation of the co-culture for SEM imaging and correlative microscopy}

The cells were fixed by incubating in $1 \% \mathrm{v} / \mathrm{v}$ glutaraldehyde (Sigma-Aldrich, Germany) in PBS at $4^{\circ} \mathrm{C}$ overnight. Then they were dehydrated by incubating for 10 minutes in increasing concentrations of ethanol $(30 \%, 40 \%, 50 \%, 60 \%, 70 \%, 80 \%, 90 \%, 96 \%$, $100 \%, 100 \%$ ), and finally chemically-dried with hexamethyldisilazane (Sigma-Aldrich). After drying, the fixed cells were sputter-coated with a gold layer (15nm approximate thickness; Q150RES; Quorum Technologies Ltd, East Grinstead, UK). In order to avoid the loss of macrophages from the apical compartment, cells from the basolateral compartment only were fixed by adding $500 \mu$ l of each reaction agent as described above.

\section{Statistics}

The results are shown as the mean \pm standard deviation (SD), and were calculated by using the Microsoft Excel ${ }^{\circledR}$ software. 


\section{Results}

\section{Establishment of the co-culture model}

Three isolations were carried out and analysed, in order to determine the optimal cell seeding density. It always proved possible to isolate at least 1 $\times 10^{6}$ cells per gramme of lung tissue. After isolation, all the epithelial cell cultures reached an appropriate TEER $\left(\sim 500 \Omega . \mathrm{cm}^{2}\right)$ on day 4 , and the TEER further increased to reach its maximum value, which was stable for at least three days. This suggested a time-frame from day 4 to day 6 when the co-culture could be set-up and the experiments performed. The maximum TEER of the epithelial cells depended on the seeding density. Therefore, seeding density was optimised by increasing the number of cells per well, reaching a plateau at approximately 200,000 cells $/ \mathrm{cm}^{2}$ (Figure 2 ). Subsequent experiments were then performed with 300,000 cells $/ \mathrm{cm}^{2}$, to ensure a robust barrier. Confocal laser microscopy showed a dense monolayer with tight junctions. It was found that the surface per cell decreased with increasing seeding density (Figure 2).

On day 4, co-cultures with the macrophages were initiated with different macrophage numbers and incubated overnight. A dense epithelial monolayer was required, because the macrophages were unable to attach to the Transwell insert. The TEER was measured on the following day, to investigate the effect of macrophages on the barrier function. In two out of three isolations, the mean TEER dropped with increasing numbers of macrophage (Figure 3): 300,000 macrophages $/ \mathrm{cm}^{2}$ reduced the TEER to below $400 \Omega . \mathrm{cm}^{2}$, which could be critical for transport studies with small molecules. For example, Steimer et al. (13) suggested a threshold level of $600 \Omega . \mathrm{cm}^{2}$ for porcine alveolar epithelial cells (13). It was obvious that the lung tissue was, in most cases, received from smokers, as evidenced by the presence of carbon-loaded macrophages. This patient-dependent unspecific staining of the macrophages was used to visualise their density by light microscopy (Figure $3)$. The macrophages were distributed on top of the epithelium in small agglomerates. Based on in vivo data reported by Stone et al. (14), we decided to use 100,000 macrophages $/ \mathrm{cm}^{2}$ for subsequent experiments. Under such conditions, the TEER of the cocultures was lower compared to that of the primary human epithelial cell (hAEpC) monolayer, but remained above $500 \Omega . \mathrm{cm}^{2}$.

\section{Deposition of airborne particles on the co-culture with the PADDOCC}

The spray-dried PLGA dry powder formulation was characterised with the NGI, to ensure that these particles would be able to reach the alveolar space in vivo. This confirmed the relevance of subsequent experiments. The FPF (i.e. particles with an aerodynamic diameter of $<5 \mu \mathrm{m}$ ), as determined with the NGI, comprised $36 \%$ of the loaded dose. The calculation of mass mean aerodynamic diameter (MMAD) and geometric standard deviation (GSD) resulted in values of $3.7 \mu \mathrm{m}$ for MMAD and $2.3 \mu \mathrm{m}$ for GSD. The particle size of the embedded PLGA nanoparticles was measured by photon correlation spectroscopy and found to be $320 \mathrm{~nm}$ (zaverage) with a Polydispersity index (PDI) of 0.17.

Prior to the deposition of FA-PLGA particles on cell cultures, their deposition on empty Snapwells in the PADDOCC was investigated. FA-PLGA particles deposited in the Snapwells were visualised by correlative microscopy, which showed particle aggregates of up to $10 \mu \mathrm{m}$ in size (Figure 4). The amount of dry powder deposited from three capsules filled with FA-PLGA particles was quantified with a fluorescence plate reader. Of the $20 \mathrm{mg}$ of powder per capsule, $60 \mu \mathrm{g} / \mathrm{cm}^{2}$ to $110 \mu \mathrm{g} / \mathrm{cm}^{2}$ were deposited in each Snapwell (surface area $1.12 \mathrm{~cm}^{2}$ ).

In order to use the PADDOCC for air-liquid experiments, the cell culture protocol was adjusted. Macrophages were placed at a high cell density

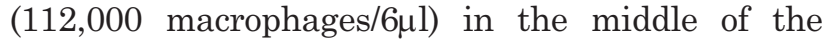
Snapwell, on epithelial cells, from where they distributed over the whole epithelial layer. Human alveolar macrophages (hAM) were seeded on day 4, and were incubated until day 5 , when the deposition of particles was carried out.

Spray-dried PLGA particles were deposited with the PADDOCC on primary autologous co-cultures (day 5). After 24 hours (day 6), TEER values were measured and an MTT assay was performed in order to detect possible cytotoxic effects of the particles. No hints of acute toxicity were detected. However, the barrier properties were clearly reduced compared to those under the submerged culture conditions (sub-

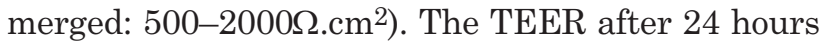
was reduced in all groups (monoculture, co-culture with and without particles), suggesting a loss of epithelial barrier function (results not shown). In two out of three isolations, no TEER could be detected independently of the experimental setting (control group or deposition). The third isolation showed the best barrier properties, with a TEER of about $400 \Omega . \mathrm{cm}^{2}$. The reduced TEER was a consequence of the change to air-liquid interface conditions. Macrophages were found in small agglomerates on top of the epithelium.

Correlative microscopy was used to investigate particle uptake into cells after the deposition with the PADDOCC. Figure 5a shows a confocal laser microscopy image where a selected region (boxed) was relocated in the SEM by using correlative microscopy (Figure 5b). The strong auto-fluorescence of the cells (red/orange) is a consequence of the glutaraldehyde fixation. Green regions indi- 
Figure 2: The assessment of monolayer integrity by using TEER measurements and immunohistochemistry

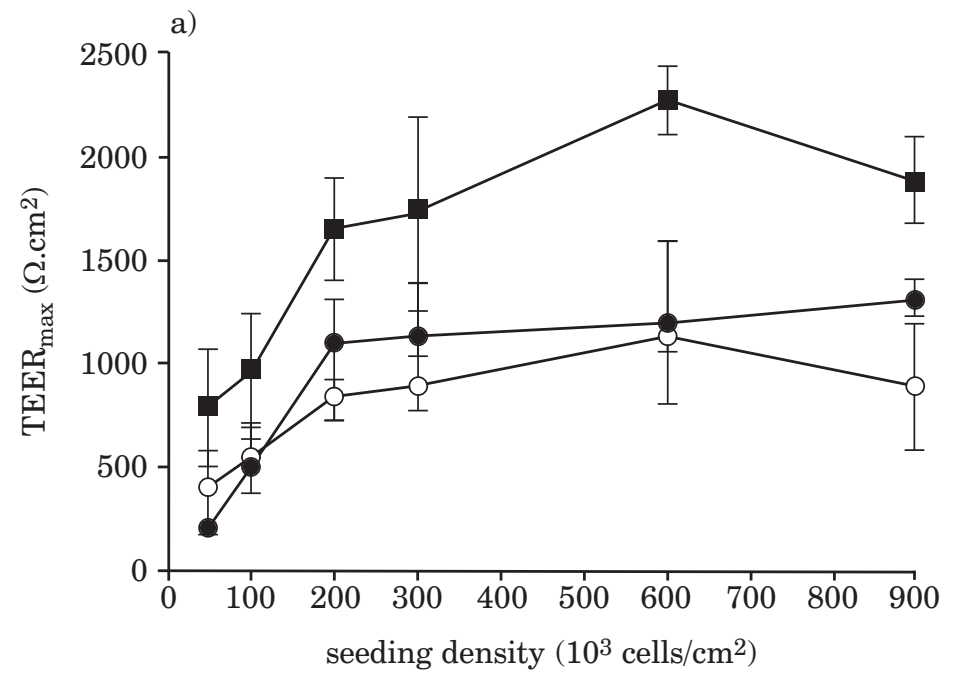

- = isolation number $1 ; 0=$ isolation number $2 ; \mathbf{\square}=$ isolation number 3.

a) Shows the maximum observed TEER $\left(T E E R_{\max }\right)$ as a function of seeding density. The TEER $R_{\text {max }}$ during culture increased up to a seeding density of $200,000 \mathrm{cells} / \mathrm{cm}^{2}$, and then tended to reach a plateau. All three isolations were performed with tissues from different donors. For each measurement, the mean of at least four Transwell inserts was determined (mean $\pm S D)$.

b)

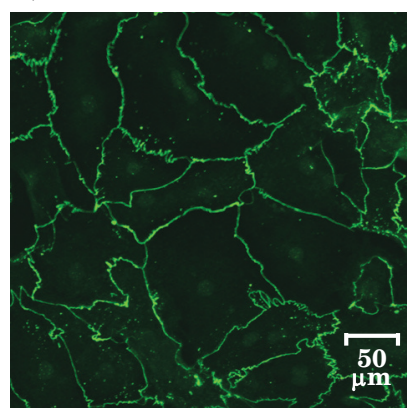

$50 \times 10^{3} \mathrm{cells} / \mathrm{cm}^{2}$

$300 \times 10^{3} \mathrm{cells} / \mathrm{cm}^{2}$

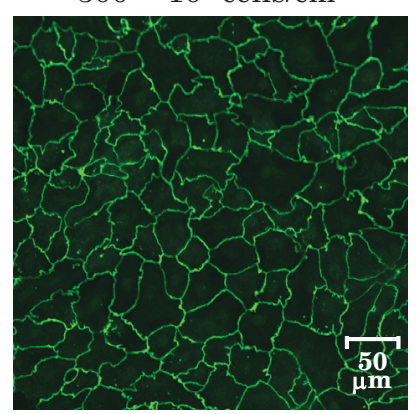

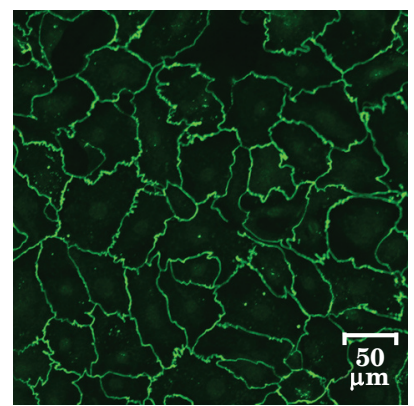

$100 \times 10^{3} \mathrm{cell} / \mathrm{s} / \mathrm{cm}^{2}$

Seeding densities

$600 \times 10^{3}$ cells $/ \mathrm{cm}^{2}$

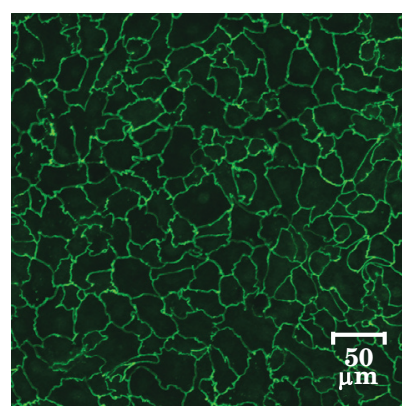

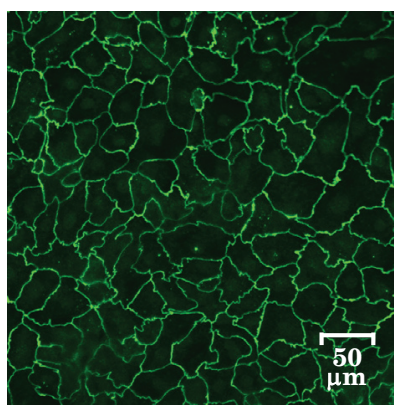

$200 \times 10^{3}$ cells $/ \mathrm{cm}^{2}$

$900 \times 10^{3}$ cells $/ \mathrm{cm}^{2}$

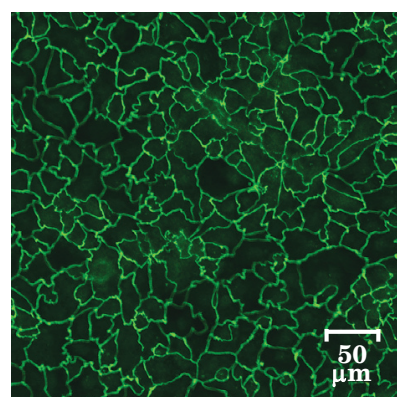

b) Shows the tight junctions of epithelial cells stained with anti-zonula occludens protein-I antibody. Tight junctions (stained with anti-occludin) were present at all seeding densities. 
Figure 3: The effects of macrophage seeding density on the TEER

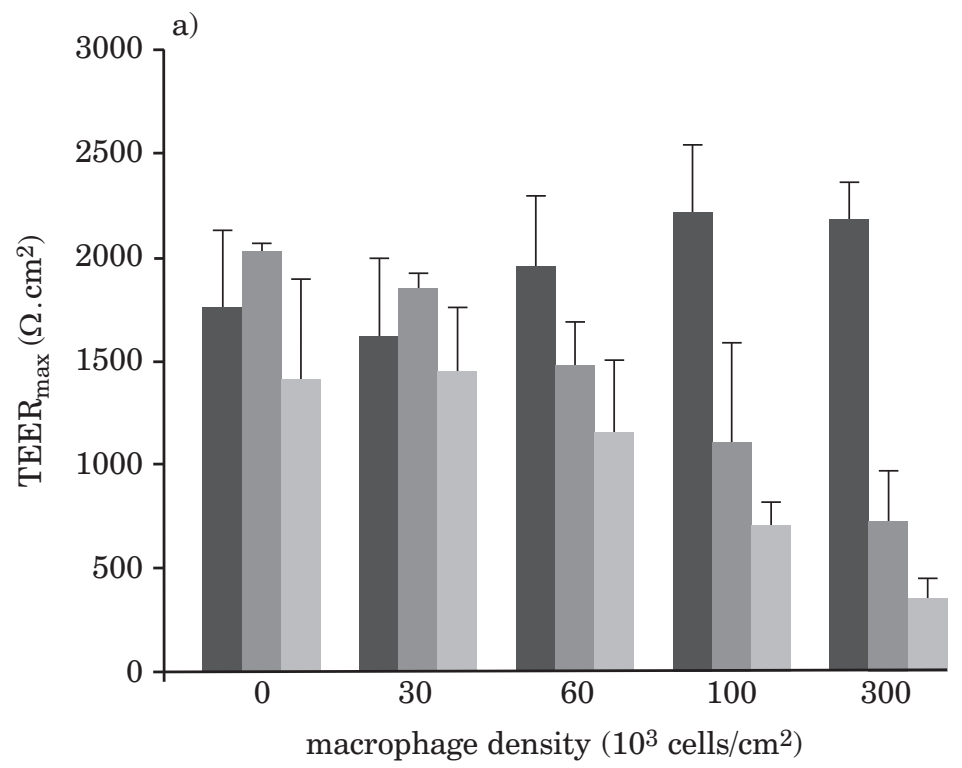

a) In two of three isolations, a reduction of the barrier properties was observed after the addition of the macrophages. All isolations were performed with tissues from different donors. For each measurement, the mean values for at least four Transwell inserts were determined (mean $\pm S D$ ).

$=$ isolation $A ; \quad=$ isolation $B ; \quad=$ isolation $C$.

b)

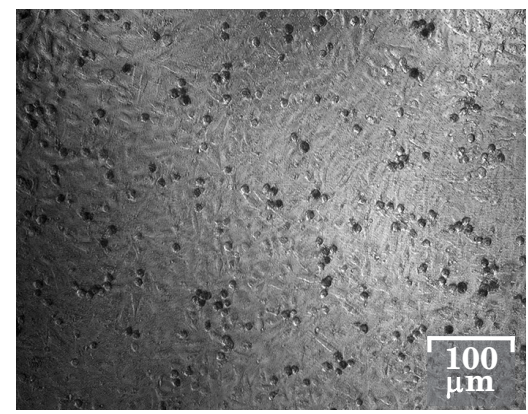

$30 \times 10^{3} \mathrm{cells} / \mathrm{cm}^{2}$

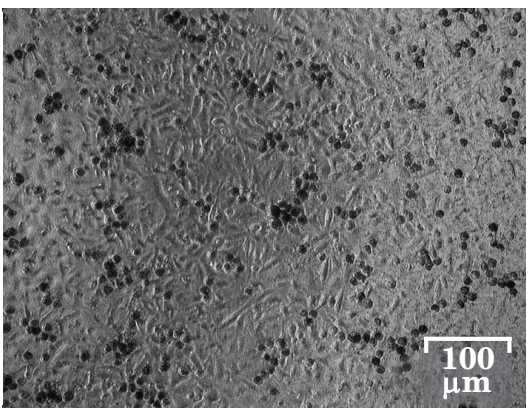

$60 \times 10^{3} \mathrm{cells} / \mathrm{cm}^{2}$

Cell numbers

$100 \times 10^{3} \mathrm{cells} / \mathrm{cm}^{2}$

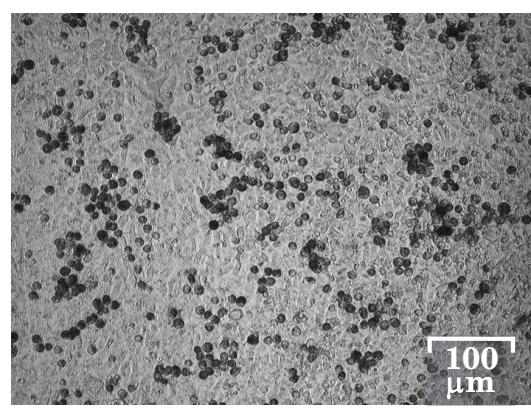

$300 \times 10^{3}$ cells $/ \mathrm{cm}^{2}$

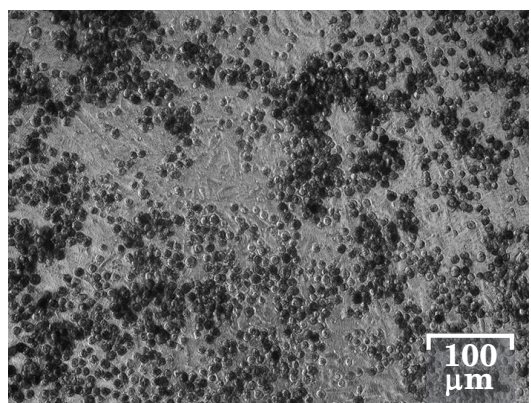

b) Different numbers of macrophages (with cell numbers as indicated) were visible on top of the epithelial cells, distributed in small agglomerates. 
cate an accumulation of particles. After 24 hours, significant amounts of particles were still observed outside the cells, as shown in the selected region, suggesting incomplete particle uptake by the macrophages (Figure 5c).

\section{Discussion}

Novel aerosol and dry powder formulations for pulmonary drug delivery need to be characterised with regard to safety and efficacy. In order to be useful, a model of the air-blood barrier has to provide a tight diffusional barrier and macrophage clearance. The autologous co-culture system was investigated with the aim of modelling the essential cellular elements of the pulmonary air-blood barrier, which are mainly supplied by alveolar epithelial cells and macrophages. Our strategy was to recombine these two cell types, after isolation from the same donor (i.e. autologous), in co-cultures.

The use of primary cells is hindered by several obstacles. Firstly, the availability of human tissue is limited, and the isolation procedure is time and cost intensive. In addition, appropriate permission from the responsible ethics committee is required.
Also, seeding densities published in the literature differ, depending on the protocol used (6, 7, 15-18). In an attempt to overcome some of these issues, our first aim was to reduce the seeding cell density per insert to the necessary minimum, which would increase the number of experiments possible. We achieved a constant TEER for epithelial cell densities > 200,000 cells $/ \mathrm{cm}^{2}$, which indicated that higher cell numbers would not lead to further improvements to the cellular barrier. However, based on our own data, we decided to use 300,000 cells $/ \mathrm{cm}^{2}$, which is slightly above the apparent critical threshold value of 200,000 cells $/ \mathrm{cm}^{2}$. Compared to other models, such as the tetra-culture system of Klein et al. that uses the cell line A549 $\left(120,000\right.$ cells $\left./ \mathrm{cm}^{2}\right)$, this seeding density for primary epithelial cells is relatively high (19). Apart from the fact that A549 cells do not express tight intercellular junctions, differences in drug permeability, as compared to other cell types, can be expected, due to the different expression of transporter proteins (18).

Particle clearance was addressed by co-culturing epithelial cells with human primary alveolar macrophages. The issue of potential macrophage activation during the isolation or culturing processes was addressed in additional experiments

\section{Figure 4: Deposition of spray-dried fluoresceinamine-labelled poly(lactid-co-glycolid) particles (green) in the PADDOCC}

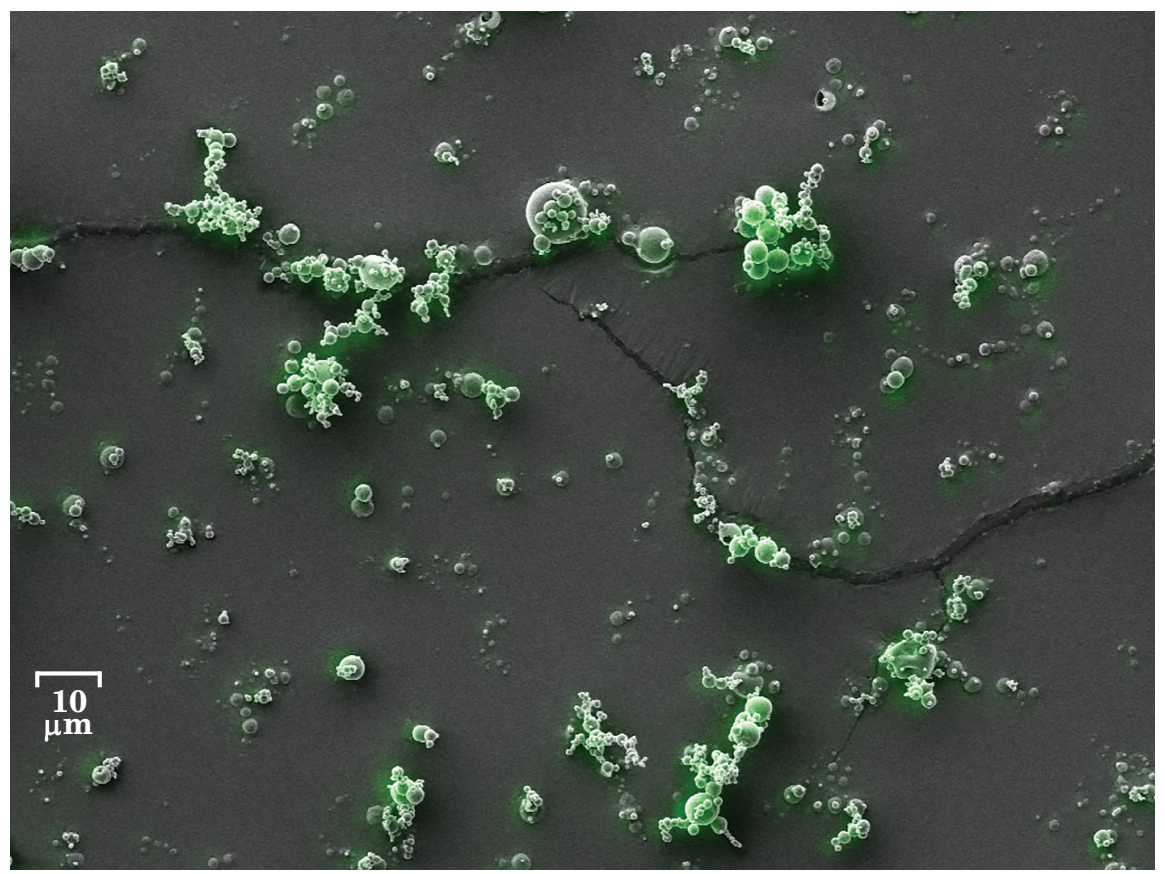

$5.00 \mathrm{kV}$

SE1

Chamber $=5.94 \mathrm{e}-004 \mathrm{~Pa}$

Width $=185.9 \mu \mathrm{m}$

EVO HD15

$8.0 \mathrm{~mm}$

$1.61 \mathrm{KX}$

Image pixel size $=181.5 \mathrm{~nm}$

Probe $=20 \mathrm{pA}$

Three capsules were each loaded with 20mg dry powder including fluoresceinamine-labelled poly(lactid-co-glycolid) and deposited in the Pharmaceutical Aerosol Deposition Device On Cell Cultures (PADDOCC). The mass of particles deposited varied from 60 to $110 \mu \mathrm{g} / \mathrm{cm}^{2}$ on $1.12 \mathrm{~cm}^{2}$. The particles were visualised with correlative microscopy (a combination of fluorescence microscopy and electron microscopy), which showed small aggregates of green particles, of up to $10 \mu \mathrm{m}$ in diameter. 


\section{Figure 5: Deposition of spray-dried PLGA particles on the co-culture, using the Pharmaceutical Aerosol Deposition Device On Cell Cultures (PADDOCC)}

a)

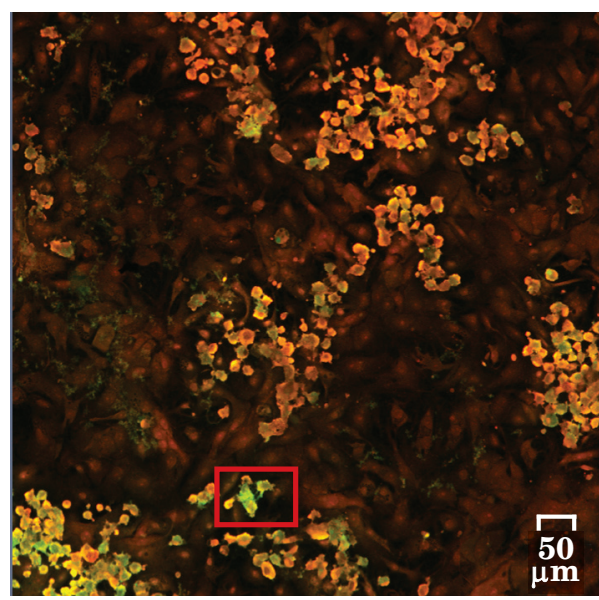

c)

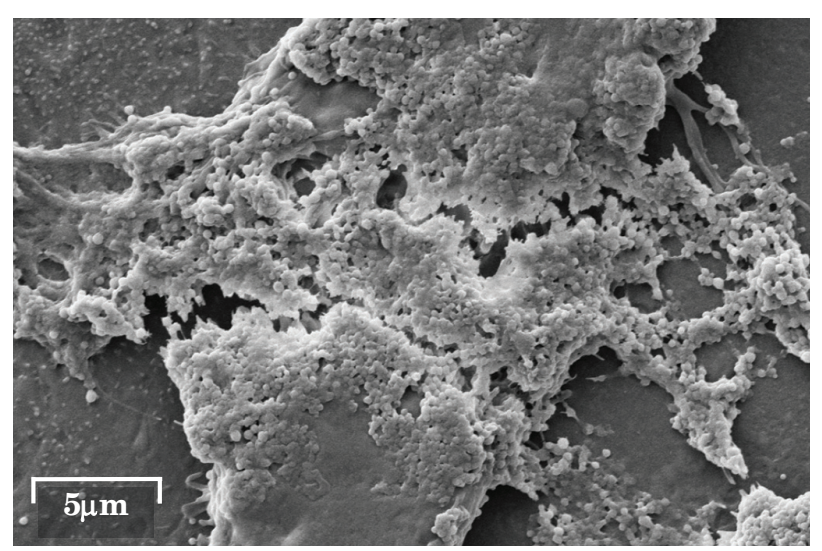

$6.00 \mathrm{kV}$ SE1 Chamber $=6.55 \mathrm{e}-004 \mathrm{~Pa}$ Width $=31.31 \mu \mathrm{m} \quad$ EVO HD15 $7.6 \mathrm{~mm}$ 9.58KX Image pixel size $=30.58 \mathrm{~nm}$ Probe $=50 \mathrm{pA}$ b)

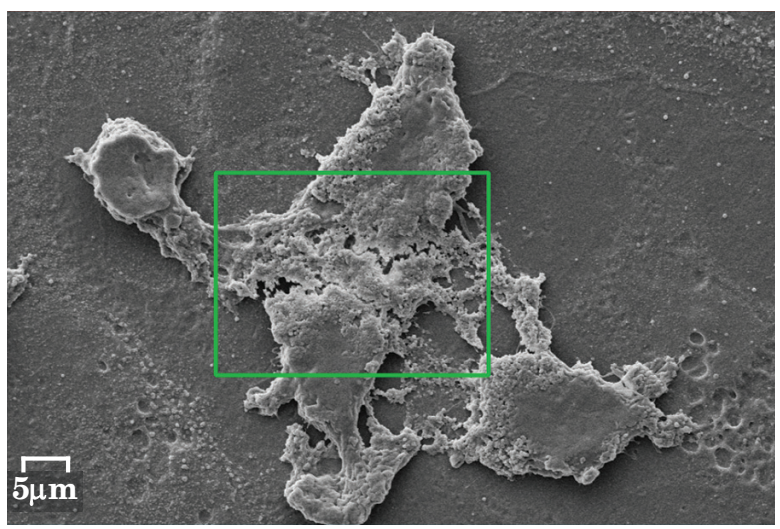

$6.00 \mathrm{kV}$ SE1 Chamber $=6.58 \mathrm{e}-004$ Pa Width $=81.49 \mu \mathrm{m} \quad$ EVO HD15 $7.6 \mathrm{~mm} \quad 3.68 \mathrm{KX}$ Image pixel size $=79.58 \mathrm{~nm} \quad$ Probe $=50 \mathrm{pA}$

a) The scanning electron microscopy (SEM) image shows macrophages spread in small aggregates on top of the epithelium after 48 hours in co-culture. The cells were fixed in glutaraldehyde and dried with increasing ethanol concentrations. Confocal laser microscopy was used to identify fluoresceinamine-labelled particles.

b) The same field was re-positioned in the SEM to visualise details with a higher resolution.

c) The higher magnification section shows that the PLGA particles stuck together and were not completely engulfed by the macrophages.

that are described in the accompanying paper (20). However, it must be noted that most of the donor lungs came from smokers, which leads to implications during imaging as a result of already internalised particles. Monocyte-derived macrophages might lead to a similar outcome, even if their phenotype differed from that of the fully-differentiated primary macrophages (8). For example, both macrophage types can be stimulated by LPS and express IL- 6 as read-out.

A reduction in TEER was observed with increasing macrophage density. A ratio of 100,000 macrophages $/ \mathrm{cm}^{2}$ to 300,000 epithelial cells is in accordance with the cell numbers reported by Stone et. al. (14). Their study showed that a human lung contains $19,600 \times 10^{6} \pm 9600 \times 10^{6}$ alveolar type I cells and $5990 \times 10^{6} \pm 1900 \times 10^{6}$ macrophages, which represents a ratio of $3: 1$. We only considered type I cells because they cover more than $90 \%$ of the lung surface, and we did not contemplate the overall cell numbers (i.e. the sum of AT I-like + AT II cells compared to macrophages). However, the chosen number of macrophages in an in vitro model is not fixed and could be adjusted in future experiments. Even if barrier properties remain acceptable up to 100,000 macrophages $/ \mathrm{cm}^{2}$, 
the TEER reduction as a consequence of the addition of the macrophages is remarkable, and might be due to two factors: a) it is possible that gaps still remain in the epithelial cell layer, allowing macrophages to prevent the formation of a tight barrier; and b) active macrophage migration could lead to a reduced TEER. The Snapwell membrane can be considered as an artificial barrier that immediately curtails any possible migratory process. As an alternative, a change from Snapwell membranes to other systems, such as the SiMPLI ${ }^{\mathrm{TM}}$ system, might lead to further insights and is closer to the overall thickness of the airblood barrier. The membranes used in this study had a thickness of $10 \mu \mathrm{m}$, whereas the SiMPLI inserts have a thinner membrane of only $0.5 \mu \mathrm{m}$.

PLGA was chosen for the first evaluation of the co-cultures, because this polymer allows the preparation of both microparticles and nanoparticles by established procedures. In addition, PLGA is characterised by an excellent biocompatibility and the possibility of tailoring its biodegradability by varying its composition (lactide/glycolide ratio), molecular weight and chemical structure (21). For a more realistic deposition of a dry powder formulation, the autologous co-culture was combined with the PADDOCC. The set-up of air-liquid interface conditions was challenging and required an adjustment of the protocol. Air-liquid conditions led to a decrease of the barrier properties compared to submerged conditions. Therefore, it is critical to investigate effects on barrier properties after 24 hours, by using the primary co-culture model. In addition, it has to be kept in mind that the dose of particles deposited per epithelial surface area is much higher than in vivo: from a $20 \mathrm{mg}$ capsule, $60 \mu \mathrm{g} / \mathrm{cm}^{2}$ to $110 \mu \mathrm{g} / \mathrm{cm}^{2}$ were deposited, while the same dose homogeneously distributed over the entire surface of the human lung $\left(\sim 100 \mathrm{~m}^{2}\right)$ would theoretically result only in $0.02 \mu \mathrm{g} / \mathrm{cm}^{2}$ (i.e. an estimate of 20mg/100m ${ }^{2}$ ). Nevertheless, 24 hours after deposition in the PADDOCC, particles were found to be associated with macrophages and in small agglomerates on the epithelial cells. This underlines the relevance of macrophages in such a model, which will be most affected by particle deposition as they are responsible for the alveolar clearance.

Human autologous co-cultures in combination with the PADDOCC appear to be a promising in vitro system that includes important aspects of the human in vivo situation. The model based on primary cells can help identify differences between cell line-based systems and primary human cells. Compared to, for example, the already established triple-culture model (22), there are still many open questions on this co-culture model. However, experiments to characterise cytokine release in the model have already been performed (23). In the accompanying paper, on the characterisation of the autologous co-culture (20), we describe how the suitability of the system as a tool for studying the efficacy of a new anti-inflammatory dry powder formulation was investigated. We show how typical inflammatory markers, such as IL-6 and TNF$\alpha$, were studied in the non-activated, activated and the treated co-cultures, in order to improve our understanding of this new in vitro system.

\section{Conclusions}

Human alveolar epithelial-macrophage co-cultures mimic the alveolar barrier, including the epithelial transport barrier and particle uptake by macrophages. The densities of epithelial cells, macrophages and the culture conditions have a strong impact on the barrier properties of this model. We conclude that the primary autologous co-culture is not suitable for high-throughput application, due to ethical issues related to the use of human tissue, the complex isolation process, and the limited amount of tissue and limited cell yield, respectively. However, in contrast to animal studies, primary human cells might improve the prediction of the in vivo clinical situation in patients. We recommend the application of the primary human autologous co-culture system as a standard for identifying similarities and differences between cell line-based models. As a consequence, the relevance of in vitro models addressing the alveolar space might improve and contribute to the reduction of the number of animal experiments and their eventual replacement.

\section{Acknowledgements}

Marius Hittinger was financially supported by the German Federal Ministry of Education and Research Project PeTrA (Support Code: 13N11458). Xabier Murgia, Petra König, Jana Westhues, Leon Muijs and Stephanie Kletting are thanked for their support and illuminating scientific discussions.

Received 12.04.16; received in final form 24.06.16; accepted for publication 30.06.16.

\section{References}

1. Patton, J.S. \& Byron, P.R. (2007). Inhaling medicines: Delivering drugs to the body through the lungs. Nature Reviews Drug Discovery 6, 67-74.

2. Hittinger, M., Juntke, J., Kletting, S., SchneiderDaum, N., de Souza Carvalho, C. \& Lehr, C-M. (2015). Preclinical safety and efficacy models for pulmonary drug delivery of antimicrobials with focus on in vitro models. Advanced Drug Delivery Reviews 85, 44-56.

3. Huh, D., Matthews, B.D., Mammoto, A., Montoya- 
Zavala, M., Hsin, H.Y. \& Ingber, D.E. (2010). Reconstituting organ-level lung functions on a chip. Science, New York 328, 1662-1668.

4. Lenz, A-G., Stoeger, T., Cei, D., Schmidmeir, M., Semren, N., Burgstaller, G., Lentner, B., Eickelberg, O., Meiners, S. \& Schmid, O. (2014). Efficient bioactive delivery of aerosolized drugs to human pulmonary epithelial cells cultured in air-liquid interface conditions. American Journal of Respiratory Cell \& Molecular Biology 51, 526-535.

5. Hein, S., Bur, M., Kolb, T., Muellinger, B., Schaefer, U.F. \& Lehr, C-M. (2010). The Pharmaceutical Aerosol Deposition Device on Cell Cultures (PADDOCC) in vitro system: Design and experimental protocol. ATLA 38, 285-295.

6. Hermanns, M.I., Fuchs, S., Bock, M., Wenzel, K., Mayer, E., Kehe, K., Bittinger, F. \& Kirkpatrick, C.J. (2009). Primary human coculture model of alveolo-capillary unit to study mechanisms of injury to peripheral lung. Cell \& Tissue Research 336, 91105 .

7. Elbert, K.J., Schäfer, U.F., Schäfers, H., Kim, K., Lee, V.H.L. \& Lehr, C. (1999). Monolayers of human alveolar epithelial cells in primary culture for pulmonary absorption and transport studies. Pharmaceutical Research 16, 601-608.

8. Hoppstädter, J., Diesel, B., Zarbock, R., Breinig, T., Monz, D., Koch, M., Meyerhans, A., Gortner, L., Lehr, C-M., Huwer, H. \& Kiemer, A.K. (2010). Differential cell reaction upon Toll-like receptor 4 and 9 activation in human alveolar and lung interstitial macrophages. Respiratory Research 11, 124.

9. Daum, N., Kuehn, A., Hein, S., Schaefer, U.F., Huwer, H. \& Lehr, C-M. (2012). Isolation, cultivation, and application of human alveolar epithelial cells. Methods in Molecular Biology 806, 31-42.

10. Weiss, B., Schaefer, U.F., Zapp, J., Lamprecht, A., Stallmach, A. \& Lehr, C-M. (2006). Nanoparticles made of fluorescence-labelled poly(L-lactide-co-glycolide): Preparation, stability, and biocompatibility. Journal of Nanoscience \& Nanotechnology 6, 30483056.

11. Hein, S., Bur, M., Schaefer, U.F. \& Lehr, C-M. (2011). A new Pharmaceutical Aerosol Deposition Device on Cell Cultures (PADDOCC) to evaluate pulmonary drug absorption for metered dose dry powder formulations. European Journal of Pharmaceutics \& Biopharmaceutics 77, 132-138.

12. Tscheka, C., Hittinger, M., Lehr, C-M., SchneiderDaum, N. \& Schneider, M. (2015). Macrophage uptake of cylindrical microparticles investigated with correlative microscopy. European Journal of Pharmaceutics \& Biopharmaceutics 95, 151-155.

13. Steimer, A., Laue, M., Franke, H., HaltnerUkomado, E. \& Lehr, C-M. (2006). Porcine alveolar epithelial cells in primary culture: Morphological, bioelectrical and immunocytochemical characterization. Pharmaceutical Research 23, 2078-2093.
14. Stone, K.C., Mercer, R.R., Gehr, P., Stockstill, B. \& Crapo, J.D. (1992). Allometric relationships of cell numbers and size in the mammalian lung. American Journal of Respiratory Cell \& Molecular Biology 6, 235-243.

15. Fuchs, S., Hollins, A.J., Laue, M., Schaefer, U.F., Roemer, K., Gumbleton, M. \& Lehr, C.M. (2003). Differentiation of human alveolar epithelial cells in primary culture: Morphological characterization and synthesis of caveolin-1 and surfactant proteinC. Cell \& Tissue Research 311, 31-45.

16. Bur, M., Huwer, H., Lehr, C-M., Hagen, N., Guldbrandt, M., Kim, K-J. \& Ehrhardt, C. (2006). Assessment of transport rates of proteins and peptides across primary human alveolar epithelial cell monolayers. European Journal of Pharmaceutical Sciences 28, 196-203.

17. Leonard, F., Collnot, E.M. \& Lehr, C-M. (2010). A three-dimensional coculture of enterocytes, monocytes and dendritic cells to model inflamed intestinal mucosa in vitro. Molecular Pharmaceutics 7, 2103-2119.

18. Salomon, J.J., Gausterer, J.C., Yahara, T., Hosoya, K., Huwer, H., Hittinger, M., Schneider-Daum, N., Lehr, C-M. \& Ehrhardt, C. (2015). Organic cation transporter function in different in vitro models of human lung epithelium. European Journal of Pharmaceutical Sciences 80, 82-88.

19. Klein, S.G., Serchi, T., Hoffmann, L., Blömeke, B. \& Gutleb, A.C. (2013). An improved 3D tetraculture system mimicking the cellular organisation at the alveolar barrier to study the potential toxic effects of particles on the lung. Particle \& Fibre Toxicology 10, 31 .

20. Hittinger, M., Mell, N-A., Huwer, H., Loretz, B., Schneider-Daum, N. \& Lehr, C-M. (2016). Autologous co-culture of primary human alveolar macrophages and epithelial cells for investigating aerosol medicines. Part II: Evaluation of IL-10loaded microparticles for the treatment of lung inflammation. ATLA 44, 349-360.

21. Ungaro, F., d'Angelo, I., Miro, A., La Rotonda, M.I. \& Quaglia, F. (2012). Engineered PLGA nano- and micro-carriers for pulmonary delivery: Challenges and promises. Journal of Pharmacy \& Pharmacology 64, 1217-1235.

22. Rothen-Rutishauser, B.M., Kiama, S.G. \& Gehr, P. (2005). A three-dimensional cellular model of the human respiratory tract to study the interaction with particles. American Journal of Respiratory Cell \& Molecular Biology 32, 281-289.

23. Haghi, M., Hittinger, M., Zeng, Q., Oliver, B., Traini, D., Young, P.M., Huwer, H., Schneider-Daum, N. \& Lehr, C-M. (2015). Mono- and cocultures of bronchial and alveolar epithelial cells respond differently to proinflammatory stimuli and their modulation by salbutamol and budesonide. Molecular Pharmaceutics 12, 2625-2632. 